\title{
Assessment of three dimensional quantitative coronary analysis by using rotational angiography for measurement of vessel length and diameter
}

\author{
Jin Bae Lee $\cdot$ Sung Gug Chang $\cdot$ So Yeon Kim • \\ Young Soo Lee $\cdot$ Jae Kean Ryu $\cdot$ Ji Yong Choi $\cdot$ \\ Kee Sik Kim · Jae Sik Park
}

Received: 8 July 2011/ Accepted: 28 November 2011

(C) The Author(s) 2011. This article is published with open access at Springerlink.com

\begin{abstract}
The aim of the study was to assess the accuracy of the three-dimensional (3D) quantitative coronary analysis (QCA) system by comparing with that of intravascular ultrasound (IVUS) QCA and twodimensional (2D) QCA. 3D QCA, 2D QCA and IVUS QCA were performed in 45 vessel segments. The obtained values for the branch to branch segment vessel length and the proximal part of the segment vessel's lumen diameter were measured. Intertechnique agreement was analyzed using paired sample $t$-test and Bland-Altman analysis. No differences were found in vessel lengths taken by 3D QCA and IVUS QCA (mean difference: $0.29 \pm 1.06 \mathrm{~mm}$, $P=0.07)$. When compared with IVUS QCA, 2D QCA underestimated vessel length (mean difference: $-1.78 \pm 2.55, P<0.001$ ). Bland-Altman analysis showed close agreement and a small bias between $3 \mathrm{D}$ QCA and IVUS QCA in the measurement of vessel
\end{abstract}

J. B. Lee - S. G. Chang - Y. S. Lee - J. K. Ryu •

J. Y. Choi · K. S. Kim

Department of Cardiology, School of Medicine,

Catholic University of Daegu, Daegu, Korea

S. Y. Kim

Department of Cardiology, School of Medicine,

Dongguk University, Gyeongju, Korea

J. S. Park (四)

Department of Physiology, School of Medicine,

Kyungpook National University, 50, Sam-Duk 2 Ga,

700-721 Jung Gu, Daegu, Korea

e-mail: jaespark@knu.ac.kr length. The vessel lumen diameter measurements by 2D QCA and 3D QCA were significantly lower than that by IVUS QCA (mean difference: $-0.64 \pm 0.69$, $P<0.001 ;-0.56 \pm 0.52, P<0.001$ respectively). Rotational angiography with $3 \mathrm{D}$ reconstruction can provide a more accurate vessel length measurement, whereas 2D and 3D QCA underestimated the vessel lumen diameter compared with IVUS QCA.

Keywords Quantitative coronary angiography · Intravascular ultrasound · Three-dimensional rotational angiography

\section{Introduction}

The main purpose of coronary angiography is to identify the coronary anatomy and the degree of luminal obstruction of the coronary arteries. In spite of its widespread use for the diagnosis and treatment of coronary artery disease, the imaging characteristics of conventional two-dimensional (2D) angiography may misrepresent and impair the accurate representation of three-dimensional (3D) vascular structures. The limitations of conventional angiographic imaging are vessel overlap, vessel foreshortening, variable magnification, bifurcation take-off angles, the difficulty to compare serial examinations and the minimal information about the vessel walls [1-5]. The accuracy of 
the quantitative coronary analysis (QCA) measurements depends on the absence of vessel overlap, minimal vessel foreshortening, obtaining similar image planes of the vessel segment and the accuracy of calibration. The position of the image intensifier and the relative magnification further confound the obtaining of accurate QCA measurements [6].

Rotational angiography is an image acquisition technique that displays vascular structures in a threedimensional (3D) like format and this technique provides significantly more visual information than conventional angiography. Intravascular ultrasound (IVUS) provides transluminal images of the coronary arteries in vivo.

The aim of this study was to assess the accuracy of the 3D QCA and two-dimensional (2D) QCA system for measuring the vessel length, the vessel diameter, as compared with that of IVUS.

\section{Subjects and methods}

Study population

The subjects were recruited from a pool of patients who were previously scheduled to undergo coronary angiography. The inclusion criteria were age above 18 years and normal renal function (as defined by a baseline serum creatinine level $<1.5 \mathrm{mg} / \mathrm{dl}$ ). Subjects were excluded if they had any of the following: previous allergies or reactions to contrast agents, complete occlusion of any coronary arteries, if they displayed atrial fibrillation on a resting ECG or if they had previously undergone coronary artery bypass surgery. Segments were excluded if of ambiguous bifurcation points of side branch origins, overlapping of vessels or severe calcified segments. 3D QCA, 2D QCA and IVUS QCA were performed in 29 patients with 45 segments (mean age: $65.7 \pm 12.2$ years, male/female ratio: 18/11). The segment of interest was defining two clearly identifiable bifurcation origins that constituted each segment by both standard angiographic views and 3D reconstruction views. Finally 45 segments were eligible for evaluation. The demographic data of the patients and characteristics of eligible vessel segments are summarized in Table 1. Approval for this study was obtained from the institutional review board of our medical center and an informed consent from all patients.
Table 1 Characteristics of the patients and vessels

\begin{tabular}{|c|c|c|}
\hline Variables & \multicolumn{2}{|l|}{ Value } \\
\hline Age (years) & \multicolumn{2}{|c|}{$65.7 \pm 12.2$} \\
\hline Gender (male/female) & \multicolumn{2}{|l|}{$18 / 11$} \\
\hline Weight (kg) & \multicolumn{2}{|l|}{$61.8 \pm 5.9$} \\
\hline Height $(\mathrm{cm})$ & \multicolumn{2}{|c|}{$162.0 \pm 12.8$} \\
\hline Ejection fraction $(\%)$ & \multicolumn{2}{|l|}{$55.4 \pm 6.7$} \\
\hline Diabetes $(\%)$ & \multicolumn{2}{|l|}{$7(24.1)$} \\
\hline Hypertension (\%) & \multicolumn{2}{|l|}{$9(31.0)$} \\
\hline Hypercholesterolemia (\%) & \multicolumn{2}{|l|}{$10(33.4)$} \\
\hline Smoking (\%) & \multicolumn{2}{|l|}{$11(37.9)$} \\
\hline Measured vessel segment (\%) & \multicolumn{2}{|l|}{45} \\
\hline LAD & $17(33.3)$ & $\begin{array}{l}\text { Proximal } 8 \text { (17.8) } \\
\text { Mid } 7(15.6) \\
\text { Distal } 2(4.4)\end{array}$ \\
\hline LCX & $15(29.6)$ & $\begin{array}{l}\text { Proximal } 7 \text { (15.6) } \\
\text { Mid } 6 \text { (13.3) } \\
\text { Distal } 2(4.4)\end{array}$ \\
\hline $\mathrm{RCA}$ & $13(30.3)$ & $\begin{array}{l}\text { Proximal } 4(8.9) \\
\text { Mid } 6(13.3) \\
\text { Distal } 3(6.7)\end{array}$ \\
\hline
\end{tabular}

Values are expressed as the number of patients and mean \pm standard deviation, $L A D$ left anterior descending artery, $L C X$ left circumflex artery, $R C A$ right coronary artery

Two-dimensional quantitative coronary analysis

Following the standard protocols of our laboratory, 2D coronary angiography was performed. The procedure was performed with a standard catheter set that included JL4 and JR4 catheters. All the angiographies were performed after patients had received $200 \mu \mathrm{g}$ of intracoronary nitroglycerin. The standard right coronary artery (RCA) angiograms usually consisted of three standard views, including the left anterior oblique (LAO) caudal view, the anteroposterior cranial view and the right anterior oblique (RAO) view. The standard left coronary artery (LCA) angiograms usually consisted of four views, such as the LAO caudal view, LAO cranial view, RAO caudal view and RAO cranial view, and these varied slightly based on the operator's preference and the anatomic variation. The 2D QCA was performed using the automated edge detection system (Pie Medical Image BV, The Netherlands). For the calibration reference, the diameter of the contrastfilled catheters that were used ranged from 5 to $7 \mathrm{Fr}$.

The vessel length was defined as the vessel segment length between the distal carina portion of the 
proximal side branch origin and the proximal carina portion of the distal side branch origin, as shown in Fig. 1. The proximal vessel diameter was measured at the point of the distal carina portion of the proximal side branch origin. Measurements were made in the frame that clearly demonstrated the proximal and distal branch origins for the vessel segment length and diameter measurements, as shown in Fig. 2.

Three-dimensional quantitative coronary analysis

The rotational angiography views were obtained after the standard views were taken and while the catheter remained engaged in the coronary artery. Proper alignment for the spin acquisition required isocentering the patient. This was accomplished by centering the

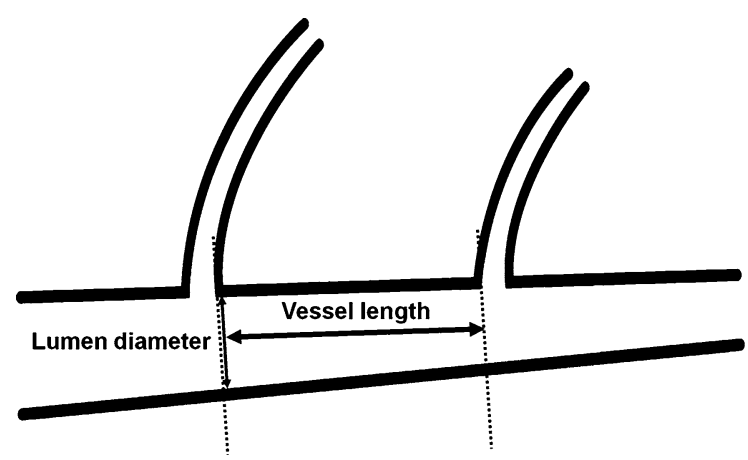

Fig. 1 The definition of vessel length and luminal diameter. The vessel length was defined as the vessel segment length between the distal carina portion of the proximal side branch origin and the proximal carina portion of the distal side branch origin (thick arrow). The proximal luminal diameter was the vessel segment diameter at the point of the distal carina portion of the proximal side branch origin (thin arrow) area of interest in the fluoroscopic field in both the anteroposterior and lateral projections. After isocentering, a test run in the spin trajectory was performed at normal speed without fluoroscopy to ensure that there was no obstruction to gantry motion. The rotation was initiated immediately after contrast was noted to fill the entire coronary artery. During spin acquisition, the gantry moved through an arc at a rate of $40 \%$ s. Cine acquisition was stopped after the contrast completely disappeared from the coronary artery. Manual injection was used for all the spin acquisitions. The LCA spins were obtained by rotating the gantry from the $30^{\circ} \mathrm{RAO} 30^{\circ}$ cranial to the left lateral position $\left(90^{\circ}\right.$ LAO $30^{\circ}$ cranial). The RCA spins started at $60^{\circ} \mathrm{LAO}$ and they were recorded through $30^{\circ}$ RAO.

The 3D QCA was performed using the automated reconstruction system (Allura 3D-CA, Phillips Medical System, The Netherlands) as shown in Fig. 3.

Intravascular ultrasound image acquisition and analysis

The IVUS studies were performed using a commercially available system (Galaxy2, Boston Scientific, Natick, MA, USA). This system incorporates a singleelement $40-\mathrm{MHz}$ transducer mounted on the tip of a flexible drive shaft within a $3.2 \mathrm{Fr}$ short monorail polyethylene imaging sheath. The transducer was withdrawn mechanically at $0.5 \mathrm{~mm} / \mathrm{s}$ with a motorized transducer pullback device to perform the imaging sequence. All the IVUS studies were performed after patients had received $200 \mu \mathrm{g}$ of intracoronary nitroglycerin. The IVUS images were recorded on a memory disc as a DICOM file for offline analysis. The IVUS catheter was advanced $5 \mathrm{~mm}$ distal to
Fig. 2 An example of the measurements by twodimensional quantitative coronary analysis. The angiographic length of a segment was determined by measuring the distance between the proximal and distal origin sites of the branches. The minimal luminal diameters were measured from the center of the stenosed lesion to the outline of the vessel wall

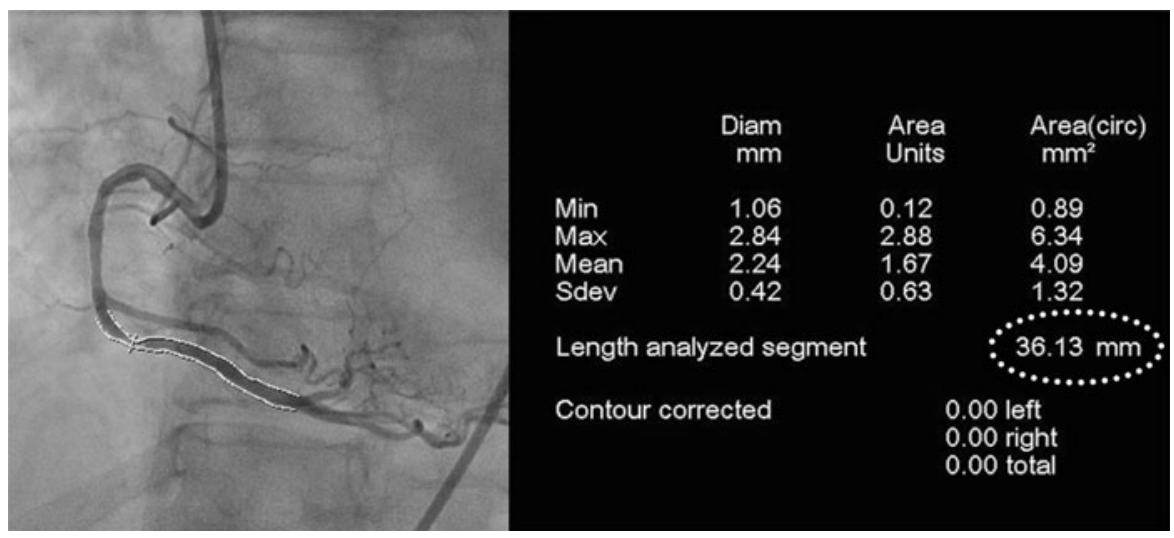


Fig. 3 An example of measurement by threedimensional quantitative coronary analysis. After rotational angiography, three-dimensional quantitative coronary analysis of the segment of interest was performed with using the automated reconstruction system

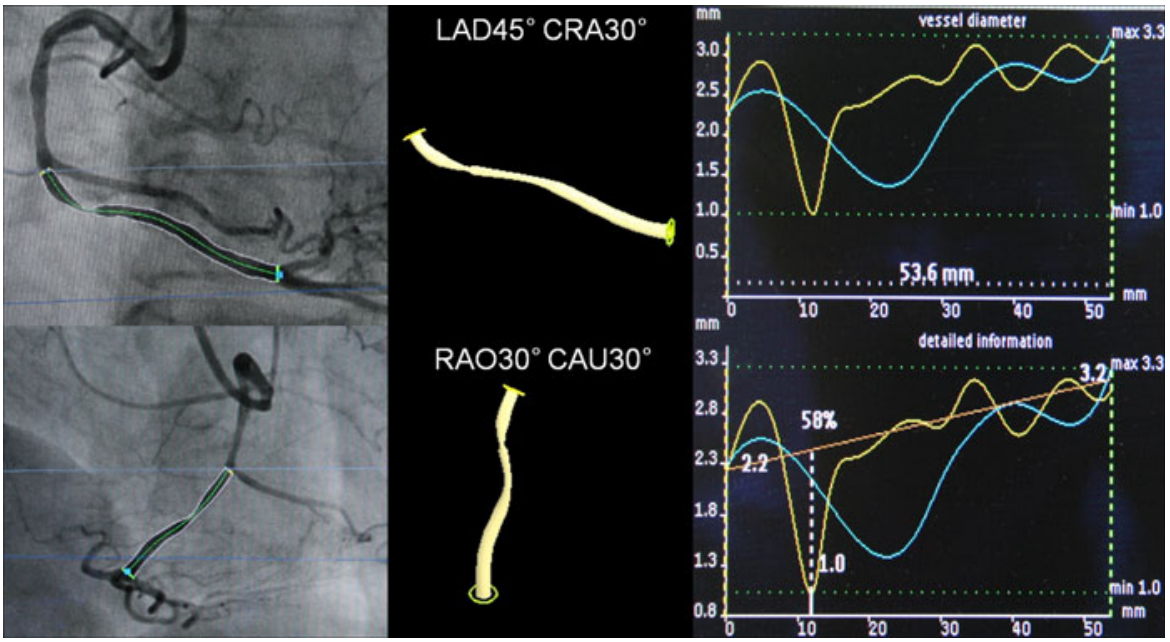

the segment site or the lesion site. The motorized transducer pullback device was activated and the imaging was then continued uninterrupted back to the aorto-ostial junction.

Quantitative analysis of the IVUS images was performed by a single individual who was kept "blinded" to the QCA. The IVUS QCA measurements were performed on an off-line computer with Echoplaque (Indec Systems, Mountain View, CA, USA). The length of the vessel segment was calculated automatically by the pullback time. The vessel lumen area was determined by tracing the intimal leading edge, and the biggest and smallest diameters were calculated automatically by software. The vessel lumen diameter was defined by means of the longest and shortest diameters.

\section{Statistics}

All the data were presented as means \pm standard deviation for continuous variables and as frequencies or percentages for the discrete variables. The obtained values of the vessel length and the vessel lumen diameter were analyzed with paired $t$-test. Pearson's correlation coefficient including its 95\% confidence interval had been calculated. BlandAltman analysis was used to further determine the agreement among the imaging modalities by calculating the bias (mean difference) and the 95\% limits of agreement (mean difference $\pm 1.96 \mathrm{SD}$ ). Statistical analysis was performed with commercially available software (SPSS for Windows, version
13.0; SPSS Inc, Chicago, IL). $P$ values $<0.05$ were considered statistically significant.

\section{Results}

Vessel length measurement

The mean value of vessel length measured by 2D QCA, 3D QCA and IVUS QCA were $21.01 \pm 9.38 \mathrm{~mm}$, $23.08 \pm 10.05 \mathrm{~mm}$ and $22.79 \pm 10.18 \mathrm{~mm}$ respectively. No significant difference was observed in vessel length measurement between 3D QCA and IVUS (paired $t$-test, $P=0.07$ ). But there was a significant difference between 2D QCA and IVUS in vessel length measurement (paired $t$-test, $P<0.001$ ) (Table 2). No significant difference was observed in vessel length measurements between 3D QCA and IVUS by each vessel territory (paired $t$-test, $P>0.05$ ). But there was a significant difference between 2D QCA and IVUS in vessel length measurement (paired $t$-test, $P<0.05$ ) (Table 3). The vessel length measured by 3D QCA was correlated with that measured by IVUS stronger than that measured by IVUS than that measured by 2D QCA (Pearson correlation; 3D QCA and IVUS: 0.99 vs. 2D QCA and IVUS: 0.97) (Table 2). Bland-Altman analysis showed that 3D QCA and IVUS QCA had good agreement with small bias in measuring the vessel length (mean bias $\pm 1.96 \mathrm{SD}, 0.29 \pm 2.09 \mathrm{~mm}$ ) and 2D QCA and IVUS QCA had moderate agreement with moderate bias in measuring vessel length (mean bias $\pm 1.96 \mathrm{SD},-1.78 \pm 5.01 \mathrm{~mm}$ ) (Fig. 4). 
Vessel lumen diameter measurement

The proximal vessel lumen diameter measured by $2 \mathrm{D}$ QCA, 3D QCA and IVUS QCA was $3.01 \pm 0.64 \mathrm{~mm}$, $3.10 \pm 0.74 \mathrm{~mm}$ and $3.66 \pm 0.88 \mathrm{~mm}$, respectively. When compared with IVUS, not only 2D QCA but also 3D QCA underestimated the vessel lumen diameter (paired $t$-test, $P<0.001$ and $P<0.001$, respectively) (Table 2). The vessel lumen diameter measurement by each vessel also underestimated both 2D QCA and 3D QCA by comparison with IVUS (Table 3). The proximal vessel lumen diameter measured by 3D QCA was correlated with that measured by IVUS stronger than that that measured by $2 \mathrm{D}$ QCA (Pearson correlation: 3D QCA and IVUS; 0.81 vs. 2D QCA and IVUS; 0.64) (Table 2). Bland-Altman analysis showed that 3D QCA and IVUS QCA had moderate agreement with moderate bias in measuring vessel lumen diameter (mean bias $\pm 1.96 \mathrm{SD}$, $-0.56 \pm 1.03 \mathrm{~mm}$ ) and 2D QCA and IVUS QCA had moderate agreement with moderate bias in measuring vessel length (mean bias $\pm 1.96 \mathrm{SD}$, $-0.64 \pm 1.35 \mathrm{~mm}$ ) (Fig. 5).

\section{Discussion}

The QCA of coronary arterial stenosis is done mainly by conventional 2D angiography; however, evaluation of the wall morphology is somewhat difficult with performing only 2D angiography [7] and the lesion morphology can be misinterpreted from the findings of conventional 2D angiography because this technique only silhouettes the vessel luminal anatomy $[8,9]$. On the other hand, IVUS enables observing a coronary arterial lumen from inside the vessel, which yields a direct image that includes the luminal diameter, the lesion length and the lesion morphology of the vessel. IVUS imaging using motorized pullback of the transducer provides reliable length measurement [10, 11]. There have been several studies that have compared the findings between 2D QCA and IVUS QCA $[3,12]$, and there is a consensus that the severity of the disease cannot accurately be determined by 2D QCA.

The measured vessel length obtained by 3D QCA revealed a higher correlation with the measured vessel length obtained by IVUS QCA than that obtained by 2D QCA. A previous study showed that 3D QCA shows a high correlation between the stent length measurement and the real stent length [13]. Vessel length was found to be significantly underestimated by 2D QCA [6]. These results demonstrate that even a quantitative method of angiography cannot accurately evaluate the length of lesions. We prospectively evaluated the vessel length, the vessel diameter and the severity of stenosis by performing 2D QCA, 3D QCA and IVUS QCA. In this study, the vessel length obtained by 3D QCA showed no significant difference from that by IVUS QCA. This means that the vessel length obtained by the 3D QCA can overcome the foreshortening of 2D QCA.

The measured vessel luminal diameters obtained by 3D QCA revealed a higher correlation to those values obtained by IVUS QCA than to those values obtained by $2 \mathrm{D}$ QCA. However, measured vessel luminal diameters obtained by both 2D and 3D QCA were less than that by IVUS QCA. This result implies that the vessel luminal diameter measured by 2D QCA and 3D QCA can underestimate the real vessel lumen diameter. There was a tendency of larger luminal diameter at reference vessel by IVUS than by 2D QCA [14]. These findings suggested 3D QCA can be used to solve the problems caused by foreshortening, however
Table 2 Paired difference of $2 \mathrm{D}, 3 \mathrm{D}$ and IVUS QCA

$S D$ standard deviation, $C I$ confidence interval, $2 D$ two-dimensional, $3 D$ threedimensional, IVUS intravascular ultrasound, $Q C A$ quantitative coronary analysis. $* P<0.001$

\begin{tabular}{lrllc}
\hline & $\begin{array}{l}\text { Mean } \pm \text { SD } \\
(\mathrm{mm})\end{array}$ & $95 \%$ CI & $\begin{array}{l}\text { Pearson's } \\
\text { coefficient }\end{array}$ & $\begin{array}{l}\text { Paired } t \text { test } \\
(P \text { value })\end{array}$ \\
\hline $\begin{array}{l}\text { Length } \\
\text { 3D-IVUS }\end{array}$ & $0.29 \pm 1.06$ & $-0.03,0.61$ & $0.99^{*}$ & 0.073 \\
3D-2D & $2.07 \pm 2.36$ & $1.36,2.78$ & $0.97^{*}$ & $<0.001$ \\
2D-IVUS & $-1.78 \pm 2.55$ & $-2.55,-1.01$ & $0.97^{*}$ & $<0.001$ \\
Diameter & & & & \\
3D-IVUS & $-0.56 \pm 0.52$ & $-0.72,-0.40$ & $0.81^{*}$ & $<0.001$ \\
3D-2D & $0.08 \pm 0.47$ & $-0.06,0.22$ & $0.78^{*}$ & 0.238 \\
2D-IVUS & $-0.64 \pm 0.69$ & $-0.85,-0.44$ & $0.64^{*}$ & $<0.001$ \\
\hline
\end{tabular}


Table 3 Paired difference of 2D, 3D and IVUS QCA by vessel

$S D$ standard deviation, $C I$ confidence interval, $2 D$ two-dimensional, $3 D$ threedimensional, IVUS intravascular ultrasound, $Q C A$ quantitative coronary analysis, $L A D$ left anterior descending artery, $L C X$ left circumflex artery, $R C A$ right coronary artery.

* $P<0.001$

\begin{tabular}{llll}
\hline $\begin{array}{l}\text { Mean } \pm \mathrm{SD} \\
(\mathrm{mm})\end{array}$ & $95 \%$ CI & $\begin{array}{l}\text { Pearson's } \\
\text { coefficient }\end{array}$ & $\begin{array}{l}\text { Paired } t \text { test } \\
(P \text { value })\end{array}$ \\
\hline
\end{tabular}

\section{$L A D$}

Length

$\begin{array}{lrcll}\text { 3D-IVUS } & 0.31 \pm 0.97 & -0.20,0.81 & 0.99^{*} & 0.216 \\ \text { 3D-2D } & 1.65 \pm 2.30 & 0.47,2.84 & 0.95^{*} & 0.009 \\ \text { 2D-IVUS } & -1.35 \pm 2.45 & -2.61,-0.88 & 0.94^{*} & 0.038\end{array}$

Diameter

$\begin{array}{lrlll}\text { 3D-IVUS } & -0.57 \pm 0.63 & -0.89,-0.25 & 0.76^{*} & 0.002 \\ \text { 3D-2D } & 0.07 \pm 0.59 & -0.23,0.37 & 0.78^{*} & 0.617 \\ \text { 2D-IVUS } & -0.64 \pm 0.88 & -1.10,-0.19 & 0.64^{*} & 0.001\end{array}$

LCX

Length

$\begin{array}{lrccc}\text { 3D-IVUS } & 0.13 \pm 1.31 & -0.60,0.85 & 0.99^{*} & 0.714 \\ \text { 3D-2D } & 2.23 \pm 2.97 & 0.59,3.87 & 0.95^{*} & 0.011 \\ \text { 2D-IVUS } & -2.10 \pm 3.27 & -3.91,-0.29 & 0.94^{*} & 0.026\end{array}$

Diameter

$\begin{array}{lrlll}\text { 3D-IVUS } & -0.39 \pm 0.44 & -0.64,-0.15 & 0.82^{*} & 0.004 \\ \text { 3D-2D } & 0.00 \pm 0.35 & -0.19,0.19 & 0.86^{*} & 0.977 \\ \text { 2D-IVUS } & -0.40 \pm 0.51 & -0.68,-0.12 & 0.75^{*} & 0.009\end{array}$

$R C A$

Length

$\begin{array}{lrccr}\text { 3D-IVUS } & 0.46 \pm 0.90 & -0.08,1.01 & 0.99^{*} & 0.090 \\ \text { 3D-2D } & 2.43 \pm 1.62 & 1.46,3.41 & 0.99^{*} & <0.001 \\ \text { 2D-IVUS } & -1.97 \pm 1.74 & -3.02,-0.92 & 0.99^{*} & 0.002\end{array}$

Diameter

\begin{tabular}{lrllr} 
3D-IVUS & $-0.74 \pm 0.43$ & $-1.00,-0.48$ & $0.80^{*}$ & $<0.001$ \\
3D-2D & $0.19 \pm 0.41$ & $-0.06,0.43$ & $0.80^{*}$ & 0.126 \\
2D-IVUS & $-0.93 \pm 0.49$ & $-1.22,-0.64$ & $0.75^{*}$ & $<0.001$ \\
\hline
\end{tabular}

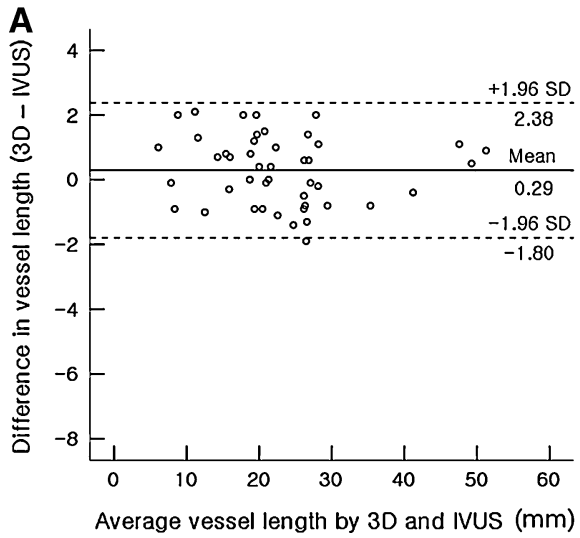

Fig. 4 Bland-Altman plots show degree of agreement between 3D QCA and IVUS (a) and 2D QCA and IVUS (b) for measurement of vessel length. Solid line indicates mean bias, and broken lines represent limit of agreement (1.96 SD). BlandAltman analysis good agreement with small bias between 3D

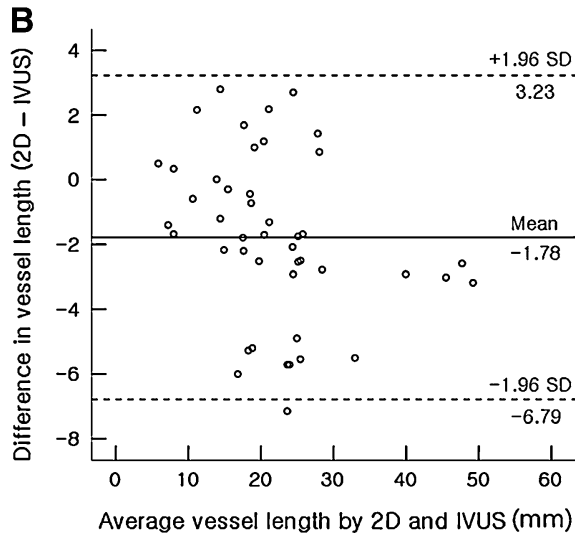

QCA and IVUS (a) and moderate agreement with moderate bias between 2D QCA and IVUS (b) in measurement of vessel length. $2 D$ two-dimensional, $3 D$ three-dimensional, IVUS intravascular ultrasound, $Q C A$ quantitative coronary analysis 


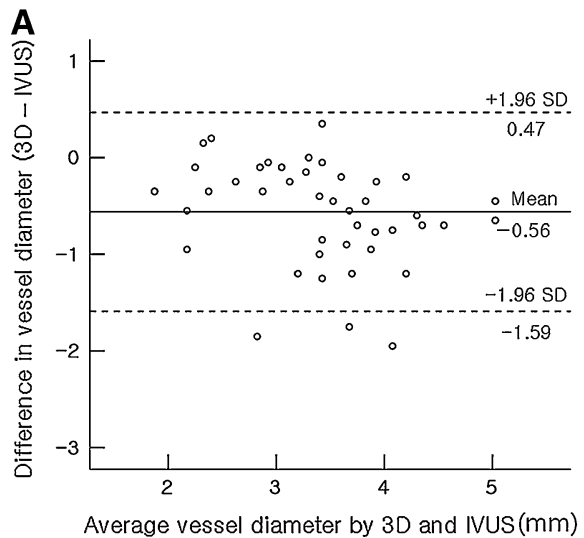

Fig. 5 Bland-Altman plots show degree of agreement between 3D QCA and IVUS (a) and 2D QCA and IVUS (b) for measurement of vessel lumen diameter. Solid line indicates mean bias, and broken lines represent limit of agreement (1.96 $\mathrm{SD})$. Bland-Altman analysis moderate agreement with moderate

major limitation of 3D system caused by luminology itself is unsolved problem in vessel diameter measurement.

The results of this study have several clinical implications, and particularly from the point of view of interventional procedures. Determining the lesion length is very important for stent deployment $[15,16]$. It has been reported that when the length was determined by 2D QCA, the selected stent did not cover the entire lesion [17]. In such cases, in-stent restenosis can occur especially at the diseased edge site. Thus, accurate determination of the lesion length is essential to perform interventional procedures properly. However, 3D QCA permits an accurate 3D geometric analysis of the coronary arteries and it is superior to 2D QCA with respect to evaluating a lesion's length. Furthermore, rotational angiography uses a standardized protocol for acquisition and the technique provides an objective and less operatordependent set of images on which to base clinical decision making. Besides, rotational coronary angiography has the potential to improve patient safety by markedly reducing the amount of radiographic contrast and radiation exposure [18].

\section{Limitation}

A relatively small number of subjects were included in this study. So the comparisons were performed using 45 segments of interest. Therefore more then one segment per vessel was selected in some cases. We

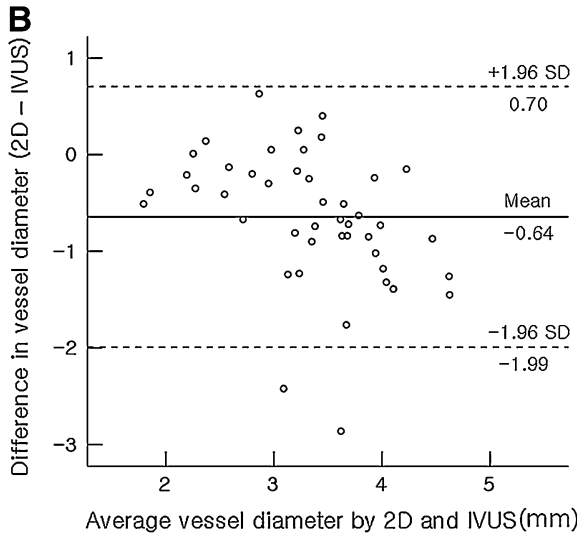

bias between 3D QCA and IVUS (a) and moderate agreement with moderate bias between 2D QCA and IVUS (b) in measurement of vessel lumen diameter. $2 D$ two-dimensional, $3 D$ three-dimensional, IVUS intravascular ultrasound, $Q C A$ quantitative coronary analysis

could not determine the real vessel length and lumen diameter of measurement, so the comparisons were done using the vessel length and lumen diameter measurements obtained via IVUS. IVUS QCA provides more accurate information as compared to $2 \mathrm{D}$ QCA but IVUS cannot provide real vessel dimensions. The vessel angulation and tortuosity could influence the results of length measurements. The vessel length measurement by IVUS can slightly underestimate the real vessel length because the IVUS catheter's path is straighter than the real curve of a blood vessel, especially for tortuous vessels. This is of minor influence on the IVUS QCA. The heavy calcification of vessel could overestimate vessel diameter by angiographic QCA, since we included noncalcified segments. The clinical applicability of that advantage of $3 \mathrm{D}$ compared to $2 \mathrm{D}$ QCA to measure segment length and determine the stent length may be limited because 3D QCA takes time and usually visual assessment is predominant in non complex lesion intervention in real world practice. The additive value of IVUS to determine vessel diameter was well validated and advantage also proved but this modality can not applicable in all patients because time and cost.

\section{Conclusion}

The use of 3D QCA of the coronary arteries during coronary intervention appears to be an attractive 
option for generating optimized view maps that can help minimize the foreshortening of the region of interest during the intervention. However, 2D and 3D QCA methods underestimated the vessel lumen diameter when compared with IVUS QCA. This study supports the accuracy of vessel length measurement and clinical applicability of the 3D QCA system.

Acknowledgments This work was supported by the grant from the Korean Institute of Medicine.

\section{Conflict of interest None.}

Open Access This article is distributed under the terms of the Creative Commons Attribution Noncommercial License which permits any noncommercial use, distribution, and reproduction in any medium, provided the original author(s) and source are credited.

\section{References}

1. Galbraith JE, Murphy ML, de Soyza N (1978) Coronary angiogram interpretation: interobserver variability. JAMA 240:2053-2056

2. Arnett EN, Isner JM, Redwood CR et al (1979) Coronary artery narrowing in coronary heart disease: comparison of cineangiographic and necropsy findings. Ann Intern Med 91:350-356

3. De Scheerder I, De Man F, Herregods MC et al (1994) Intravascular ultrasound versus angiography for measurement of luminal diameters in normal and diseased coronary arteries. Am Heart J 127:243-251

4. Mintz GS, Painter JA, Pichard AD et al (1995) Atherosclerosis in angiographically "normal" coronary artery reference segments: an intravascular ultrasound study with clinical correlations. J Am Coll Cardiol 25:1479-1485

5. Green NE, Chen SY, Messenger JC et al (2004) Threedimensional vascular angiography. Curr Probl Cardiol 29:104-142

6. Koning G, Hekking E, Kemppainen JS et al (2001) Suitability of the Cordis stabilizer marker guide wire for quantitative coronary angiography calibration: an in vitro and in vivo study. Catheter Cardiovasc Interv 52:334-341
7. Nakatani S, Yamagishi M, Tamai J et al (1995) Assessment of coronary artery distensibility by intravascular ultrasound: application of simultaneous measurements of luminal area and pressure. Circulation 91:2904-2910

8. Nissen SE, Gurley JC, Grines CL et al (1991) Intravascular ultrasound assessment of lumen size and wall morphology in normal subjects and patients with coronary artery disease. Circulation 84:1087-1099

9. Ozaki Y, Violaris AG, Kobayashi T et al (1997) Comparison of coronary luminal quantification obtained from intracoronary ultrasound and both geometric and videodensitometric quantitative angiography before and after angioplasty and directional atherectomy. Circulation 96:491-499

10. Fuessl RT, Mintz GS, Pichard AD et al (1996) In vivo validation of intravascular ultrasound length measurements using a motorized transducer pullback system. Am J Cardiol 77:1115-1118

11. Tanaka K, Carlier SG, Mintz GS et al (2007) The accuracy of length measurements using different intravascular ultrasound motorized transducer pullback systems. Int J Cardiovasc Imaging 23:733-738

12. Iwami T, Fujii T, Miura T et al (1996) Limitations of intravascular ultrasound for the evaluation of coronary luminal area: comparison with quantitative angiography. Jpn Circ J 60:575-584

13. Rainer G, Klara M, Gunter B et al (2006) Clinical assessment of a new real time 3D quantitative coronary angiography system: evaluation in stented vessel segments. Catheter Cardiovasc Interv 68:44-49

14. Kim YH, Hong MK, Kim JW et al (1997) Comparison between intravascular ultrasound and quantitative coronary angiographic measurements in coronary artery stenting. Korean Circ J 27:1265-1271

15. Mauri L, O’Malley AJ, Cutlip DE et al (2004) Effects of stent length and lesion length on coronary restenosis. Am J Cardiol 93:1340-1346

16. Grewal J, Ganz P, Selwyn A et al (2001) Usefulness of intravascular ultrasound in preventing stenting of hazy areas adjacent to coronary stents and its support of spot-stenting. Am J Cardiol 87:1246-1249

17. Gollapudi RR, Valencia R, Lee SS et al (2007) Utility of three-dimensional reconstruction of coronary angiography to guide percutaneous coronary intervention. Catheter Cardiovasc Interv 69:479-482

18. Mateen A, Kalpesh TV, Jacob M et al (2005) Randomized study of the safety and clinical utility of rotational versus standard coronary angiography using a flat-panel detector. Catheter Cardiovasc Interv 66:43-49 